\title{
Closing the light gluino gap with electron-proton colliders
}

\author{
David Curtin* \\ The Department of Physics, University of Toronto, 60 Saint George Street, \\ Toronto, Ontario M5S 1A7, Canada \\ Kaustubh Deshpande ${ }^{\dagger}$ \\ Maryland Center for Fundamental Physics, Department of Physics, \\ University of Maryland, College Park, Maryland 20742-4111, USA \\ Oliver Fischer \\ Institute for Nuclear Physics (IKP), Karlsruhe Institute of Technology, \\ Hermann-von-Helmholtz-Platz 1, D-76344 Eggenstein-Leopoldshafen, Germany \\ José Zurita ${ }^{\S}$ \\ Institute for Nuclear Physics (IKP), Karlsruhe Institute of Technology, \\ Hermann-von-Helmholtz-Platz 1, D-76344 Eggenstein-Leopoldshafen, Germany \\ and Institute for Theoretical Particle Physics (TTP), Karlsruhe Institute of Technology, \\ Engesserstraße 7, D-76128 Karlsruhe, Germany \\ (Received 3 January 2019; published 12 March 2019)

\begin{abstract}
The future electron-proton collider proposals, $\mathrm{LHeC}$ and $\mathrm{FCC}$-he, can deliver $\mathcal{O}(\mathrm{TeV})$ center-of-mass energy collisions, higher than most of the proposed lepton accelerators, with $\mathcal{O}\left(\mathrm{ab}^{-1}\right)$ luminosity, while maintaining a much cleaner experimental environment as compared to the hadron machines. This unique capability of $e^{-} p$ colliders can be harnessed in probing beyond the Standard Model scenarios giving final states that look like hadronic noise at $p p$ machines. In the present study, we explore the prospects of detecting such a prompt signal having multiple soft jets at the LHeC. Such a signal can come from the decay of gluino in $R$-parity-violating or stealth supersymmetry, where there exists a gap in the current experimental search with $m_{\tilde{g}} \approx 50-70 \mathrm{GeV}$. We perform a simple analysis to demonstrate that, with simple signal selection cuts, we can close this gap at the $\mathrm{LHeC}$ at the $95 \%$ confidence level, even in the presence of a reasonable systematic error. More sophisticated signal selection strategies and detailed knowledge of the detector can be used to improve the prospects of signal detection.
\end{abstract}

DOI: 10.1103/PhysRevD.99.055011

\section{INTRODUCTION}

The search for new physics that can address fundamental problems in the Standard Model (SM) like the hierarchy problem, dark matter, baryon asymmetry etc., depends crucially on the development of experiments capable of probing new regimes. Colliders searching for physics beyond the Standard Model (BSM) form a major and important part of such experiments. Hadron or $p p$

\footnotetext{
*dcurtin@physics.utoronto.ca

†ksd@umd.edu

*oliver.fischer@kit.edu

§ose.zurita@kit.edu
}

Published by the American Physical Society under the terms of the Creative Commons Attribution 4.0 International license. Further distribution of this work must maintain attribution to the author(s) and the published article's title, journal citation, and DOI. Funded by SCOAP ${ }^{3}$. colliders, like the Large Hadron Collider (LHC) or the future colliders FCC-hh [1-3] and SppC [4], with their enormous center-of-mass energies and luminosities, provide increasing capability to probe physics at higher mass scales, or very rare processes. The future proposed lepton or $e^{+} e^{-}$colliders like ILC [5,6], FCC-ee [7], CEPC [8,9], and CLIC [10], although having much lower center-ofmass energies than the $p p$ colliders, due to very low backgrounds, are ideal for precision measurements, in particular of Higgs physics.

The electron-proton $\left(e^{-} p\right)$ colliders are a hybrid of $e^{+} e^{-}$ and $p p$ machines. HERA, the only $e^{-} p$ collider built so far, via deep inelastic scattering (DIS) measurements, provided information about the parton distribution functions (PDFs). However, due to its lower center-of-mass energy $(320 \mathrm{GeV})$ and luminosity $\left(\sim 500 \mathrm{pb}^{-1}\right)$, it was outclassed in almost all BSM searches by Tevatron $\left(1.96 \mathrm{TeV}\right.$ and $\left.10 \mathrm{fb}^{-1}\right)$, except in the case of specific leptoquark scenarios [11,12]. The 
future proposals for $e^{-} p$ colliders - the Large Hadron electron Collider ( $\mathrm{LHeC})$ [13-15] and the Future Circular hadron-electron Collider (FCC-he) [16] —are thought of as an add-on or upgrade to their corresponding $p p$ counterparts, which hence can be installed at a much lower cost (almost an order of magnitude less) than that of the $p p$ colliders, while providing invaluable information on the structure of the proton and the Higgs and top sectors.

The unique feature of these future $e^{-} p$ colliders is that their proposed center-of-mass energies (1.3 and $3.5 \mathrm{TeV}$ at the $\mathrm{LHeC}$ and FCC-he, respectively) are much higher than that of most of the proposed lepton colliders, along with $\sim 1 \mathrm{ab}^{-1}$ total luminosity, while maintaining a clean experimental environment with much less QCD background and pileup as compared to the hadron colliders. Thus, apart from doing more precise measurements of PDFs, this capability of $e^{-} p$ colliders can also be harnessed in searches for new physics. This can be particularly useful in probing BSM scenarios which provide signal final states that look like hadronic noise at the $p p$ colliders, thus making them very hard to probe. Reference [17] studied such "stealth" signals that arise in the form of long-lived particles, which can be an essential component of various BSM scenarios and hence theoretically very well motivated. (See Ref. [18] for a review.) However, these "stealth" signals can also be prompt. Soft multijet final states with no appreciable missing transverse energy (MET) at $p p$ colliders, which we will focus on in the current study, are a prime example of this.

In Sec. II, we briefly review how such a soft multijet signal can arise from light gluino decay in $R$-parityviolating (RPV) or stealth supersymmetry (SUSY). Here we also describe the existing gap in the experimental search for such light gluinos. In Sec. III, we review the salient features of $e^{-} p$ colliders, especially the LHeC. We study light RPV gluinos at the $\mathrm{LHeC}$ in Sec. IV. We find that in the clean environment of the $\mathrm{LHeC}$, the soft multijet signal from these light gluinos can be observed at the $95 \%$ confidence level, even in the presence of a reasonable systematic error, by making use of simple signal selection cuts. Thus, the existing gap in the search for these light gluinos can be closed with the LHeC. We conclude in Sec. V.

\section{LIGHT GLUINOS}

Gluinos are a prediction of supersymmetric models [19] and are expected to have a mass below a few $\mathrm{TeV}$ if the little hierarchy problem is addressed by weak- or $\mathrm{TeV}$-scale top squarks in the spectrum. The LHC results exclude the possibility of a gluino with mass $\lesssim 2 \mathrm{TeV}$, for particular decay paths for a gluino decaying to multiple jets with a missing energy signal $[20,21]$. A more general analysis of the LHC data allowing for almost any decay path puts this lower limit at $\sim 1 \mathrm{TeV}$ [22]. The minimal supersymmetric SM gluino is bound to decay into SM particles and missing energy due to the $R$ parity.
However, if the gluino has all-hadronic multijet decay with no appreciable missing transverse energy, then the above bounds do not apply. Such a decay, e.g., $\tilde{g} \rightarrow j j j$, can come from RPV [23] or stealth SUSY [24] models. In the RPV SUSY models, this decay can happen via an intermediate heavy squark decaying to two quarks (via the RPV coupling) with no invisible particle in the final state: $\tilde{g} \rightarrow q \tilde{q} \rightarrow q q q$. In the case of stealth SUSY, the lightest supersymmetric particle (gravitino or axino) can carry away a very small amount of invisible energy, possibly below the detector resolution. This is due to the near-degenerate mass spectrum of the new SM-singlet particles $(S, \tilde{S})$ below the gluino mass. Thus the gluino decay here, $\tilde{g} \rightarrow g \tilde{S} \rightarrow$ $g S \tilde{G} \rightarrow g j j \tilde{G}$, can mimic a prompt $\tilde{g} \rightarrow j j j$ decay.

If the gluino is very light, along with having a prompt decay to multiple jets without any MET, this soft multijet signal suffers from very large QCD backgrounds and is extremely challenging to trigger on at $p p$ colliders. Indirect searches can provide important information in this regime. The analysis of LEP event shape data excluded $m_{\tilde{g}} \leq 51 \mathrm{GeV}$, independent of the decays of the gluino [25]. A similar analysis [26] using high-precision jet data from ATLAS constrained the gluino to have a mass above a few hundred $\mathrm{GeV}$. However, direct searches in this low mass range are still highly motivated, since indirect searches at $p p$ colliders are sensitive to PDFs and other effects. Direct bounds provided by the LHC for such gluinos apply only for $m_{\tilde{g}} \geq 100 \mathrm{GeV}$ [27-29] while the lowest mass reach from direct searches of $m_{\tilde{g}}=77 \mathrm{GeV}$ was provided by $\mathrm{CDF}$ detector at Tevatron [30]. As shown in Ref. [31], even the data from the UA2 detector at the SPS collider is not able to close the gap from 51 to $76 \mathrm{GeV}$ between the LEP indirect constraints and the direct $p p$ mass limit.

\section{ELECTRON-PROTON COLLIDER BASICS}

In this section, we briefly review the important features of the proposed electron-proton colliders, which are relevant for the current study. Electron-proton colliders are hybrids of $e^{+} e^{-}$and $p p$ colliders. Although they are usually considered only in the context of deep inelastic scattering measurements, they can also provide a unique advantage over $e^{+} e^{-}$and $p p$ colliders in many searches of BSM physics; see for instance Ref. [32]. The LHeC [13-15] is one of the future proposed $e^{-} p$ colliders at CERN. It will have a $7 \mathrm{TeV}$ proton beam of the high-luminosity LHC colliding with a $60 \mathrm{GeV}$ electron beam generated from a separate electron accelerator (linac). Running concurrently with the HL-LHC, the LHeC will have a center-of-mass energy of $\sim 1.3 \mathrm{TeV}$ with a total luminosity of $\sim 1 \mathrm{ab}^{-1}$ over its entire lifetime of $\sim 10$ years. At the collision point in the HL-LHC tunnel, the LHeC detector will be installed. It will be a general-purpose detector accommodating for the asymmetric nature of $e^{-} p$ collisions. The $p_{T}$ threshold to reconstruct jets at the $\mathrm{LHeC}$ is as small as $10 \mathrm{GeV}$, provided that the total energy is above $20 \mathrm{GeV}$ [14]. 
The proposal for the Future Circular Collider (FCC) at CERN also includes the FCC-he [16] which will be an even more powerful $e^{-} p$ collider. Here, the $\mathrm{LHeC}$ electron beam will collide with the $50 \mathrm{TeV}$ FCC proton beam forming a collision with a center-of-mass energy of about $3.5 \mathrm{TeV}$ and total luminosity of about $1 \mathrm{ab}^{-1}$. In this work, we will focus on the analysis at the $\mathrm{LHeC}$ since it can be installed as early as 2023, while the FCC may operate in a more distant future.

The $\mathrm{LHeC}$ will have very low pileup and QCD background as compared to the LHC. Thus it can potentially provide a unique advantage in the searches of BSM scenarios which give rise to final states with multiple soft jets that have no distinctive feature (e.g., no leptons, little missing energy, no activity in the muon system) from pure QCD processes ("hadronic noise") at $p p$ colliders. Furthermore, its large center-of-mass energy and luminosity can provide access to higher mass scales than most of the proposed $e^{+} e^{-}$colliders. This capability of $e^{-} p$ colliders in the case of long-lived particles with soft decay products and/or very short lifetimes (up to $c \tau \sim \mu \mathrm{m}$ ) was demonstrated in Ref. [17]. In the current study, we examine the potential of the $\mathrm{LHeC}$ to observe a prompt signal with multiple soft jets, which looks like hadronic noise at $p p$ colliders thus making it very hard to observe. As mentioned in the previous section, such a signal can come from the prompt decays of light RPV gluinos.

\section{PROBING LIGHT RPV GLUINOS AT THE LHeC}

\section{A. Signal}

The production of RPV gluinos is simulated in MADGRAPH5_AMC@NLO [33] at parton-level using the RPVMSSM model [34]. We perform a simple parton-level study which is sufficient to robustly demonstrate sensitivity, leaving more sophisticated studies for future work. In an $e^{-} p$ collision, gluinos are pair produced mostly via the gluon radiated off the initial- or final-state quark. Each gluino then undergoes RPV decay to $j j j$ via an intermediate heavy squark (see Fig. 1). The final state thus has seven jets, including the jet from the incoming parton. Additional processes that would be included in a more complete hadron-level study include diagrams with one or more extra jets in the final state compared to Fig. 1. This would increase the signal cross section by $\sim 30 \%$ as well as increase the signal acceptance in our analysis. As a result, our results should be regarded as a conservative reach estimate for the $\mathrm{LHeC}$.

In this paper, we will consider two benchmark values of the gluino masses in the light gluino gap as discussed in Sec. II: 50 and $70 \mathrm{GeV}$. Note that since the production cross section is fully determined by the quantum numbers of the gluino, there are no additional free parameters. ${ }^{1}$ The

\footnotetext{
${ }^{1}$ We will ignore the potential $R$-parity-conserving decays of gluinos into gravitinos, but these will in any case be very small due to the existing bounds.
}

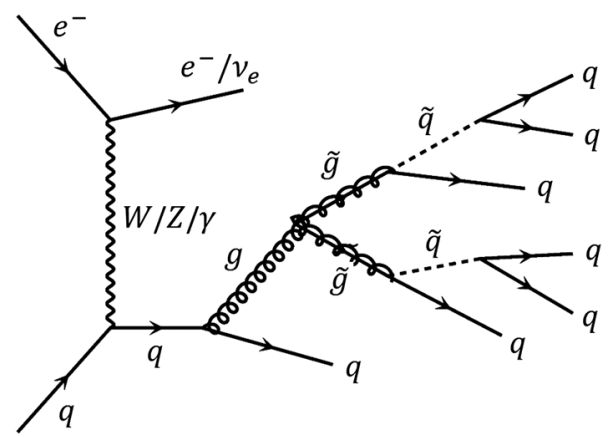

FIG. 1. Pair production of gluinos at the LHeC followed by RPV decay to $j j j$.

corresponding $\mathrm{LHeC}$ cross sections, for events with one (zero) electron in the final state, for these points are 38 (14) and 12 (4.7) fb at the leading-order parton level, respectively. For the multijet final state from gluino decays, we use the following basic generator-level cuts for jet reconstruction:

$$
p_{T}>20 \mathrm{GeV}, \quad \eta<5, \quad \Delta R>0.4 .
$$

$\Delta R>0.4$ is imposed on all possible pairs of jets in an event by manually merging the parton-level jets which are too close to each other. We remark that these are conservative choices which can be relaxed if needed; for instance, one could use $p_{T}>10 \mathrm{GeV}$ provided that $E>20 \mathrm{GeV}$, or cluster jets with smaller radii.

After applying these generator-level cuts, we are left with mostly four or five final-state jets in an event, as can be seen from Fig. 2. Hence, the seven-jet signal event from the gluino pair is effectively detected mostly as a four- or fivejet event. This is mostly a consequence of the simple assumptions on jet reconstruction we have made in our parton-level study: a full detector-level analysis with more sophisticated jet substructure techniques would likely have access to much more information on the full jet multiplicity, making our reach estimates conservative. However, this reduced effective jet multiplicity also makes it possible to estimate the SM QCD background with standard Monte Carlo techniques, as we now discuss.

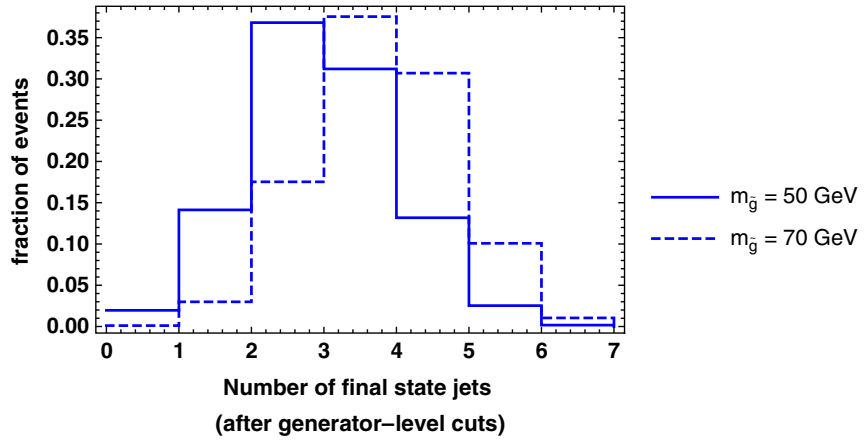

FIG. 2. Distributions for the number of final-state jets in signal events. 

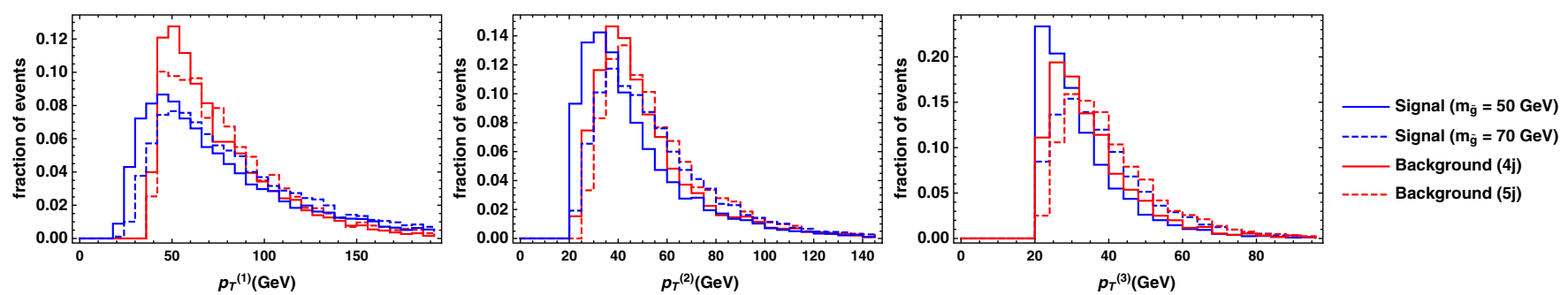

FIG. 3. $p_{T}$ distributions for the three hardest jets for the signal and background events [after applying the generator-level cuts in Eq. (1)]. The blue solid and dashed lines refer to the signal from $m_{\tilde{g}}=50$ and $70 \mathrm{GeV}$, respectively. The red solid and dashed lines refer to the QCD background with four and five jets, respectively.

\section{B. Estimating SM background and signal significance}

The dominant source of SM background for this multijet final state obviously comes from QCD processes. The treelevel estimates for the cross section for these signal processes at the $\mathrm{LHeC}$ for one (zero) electron in the final state is 5.3 $(0.27) \mathrm{pb}$ and $0.58(0.17) \mathrm{pb}$ for four-jet and five-jet events, respectively. This is obtained at the parton level using the same generator-level cuts for jets as in Eq. (1). Looking at the $p_{T}$ distribution of the jets, both for the signal and QCD background events (see Fig. 3), we consider using the signal selection cuts as defined in Table I. ${ }^{2}$

The signal and SM background cross sections after applying the cuts from Table I are listed in Table II, separately for events with one and zero electrons in the final state. As expected, the non-QCD SM background processes are quite subdominant compared to the $\mathrm{QCD}$ processes. We compute the signal significance for the two types of events, with one and zero electrons in the final state, separately as $\sigma_{1 e}$ and $\sigma_{0 e}$. The combined signal significance is obtained by adding these two in quadrature: $\sigma_{\text {net }}=\sqrt{\sigma_{1 e}^{2}+\sigma_{0 e}^{2}}$. The combined signal significance neglecting systematic effects $(S / \sqrt{B})$ of $\geq 5 \sigma$ can be achieved both for 50 and $70 \mathrm{GeV}$ gluinos using the above-mentioned cuts, as can be seen from Table III. Hence, in the absence of any systematic error, these light RPV gluinos can manifest themselves via excesses in the multijet events with $\geq 5 \sigma$ significance at the $\mathrm{LHeC}$.

Our analysis cuts are designed to be simple and robust while still yielding $5 \sigma$ statistical significance for the gluino signal. This is important since a realistic analysis will have to contend with a non-negligible systematic error, requiring not only reasonable $S / \sqrt{B}$ but also sufficient $S / B$. We now demonstrate the robustness of this search once systematic errors are taken into account. A $1 \%$ systematic error is an achievable and reasonable goal, given the proposed design of the LHeC detector [14], the possibility of data-driven background estimates in a full analysis, and progress on theoretical predictions for backgrounds. We consider the

\footnotetext{
${ }^{2}$ The low- $p_{T}$ threshold for jets at the $\mathrm{LHeC}$ of $10 \mathrm{GeV}$ can be used to apply even more efficient signal selection cuts than here, based on the distributions in Fig. 3.
}

following simplified expression for the signal significance in the presence of a systematic error $(\lambda)$ :

$$
\text { Significance }=\frac{S}{\sqrt{B+(\lambda B)^{2}}}
$$

with $\lambda=1 \%$. In the large-statistics limit, this tends to $S / \lambda B$ in the "systematics-limited" regime, justifying our cuts that also maximize $S / B$. As can be seen from Table III, the signal significance is greatly reduced after taking into account the systematic error. For instance for Cut 3 which is the strongest cut, the significance goes down from $\gtrsim 6 \sigma$ to $\lesssim 1.5 \sigma$, for a gluino mass of either 50 or $70 \mathrm{GeV}$. Hence, this calls for an additional signal selection strategy to account for the systematic error.

The signal processes involve harder momentum transfers than the corresponding QCD processes which form the dominant SM background. Hence the $p_{T}$ imparted to the outgoing $e^{-} / \nu_{e}$ in the signal processes is slightly greater than that of the background. (See Fig. 4.) Also, the signal jets are expected to be more isotropic than the QCD background jets, since they are generated from gluino decays. This implies that the maximum angular separation between a pair of jets in a signal event will be slightly less than that in a background event. (See Fig. 5.) Following these simple observations, we can employ the following additional cuts:

$$
p_{T}\left(e^{-}\right) \quad \text { or } \mathrm{MET}>p_{T}^{\min }, \quad \Delta R_{\max }^{\mathrm{jet}}<\Delta R^{*} .
$$

The first cut corresponds to $p_{T}\left(e^{-}\right)>p_{T}^{\min }$ for events with one $e^{-}$in the final state and MET $>p_{T}^{\min }$ for events with no $e^{-}$in the final state. The second cut refers to the maximum $\Delta R$ among all pairs of jets in an event being less than $\Delta R^{*}$.

The optimal use of these cuts can be made with $p_{T}^{\min } \approx$ $140 \mathrm{GeV}$ and $\Delta R^{*} \approx 3.2$. The cross sections for one (zero)

TABLE I. Signal selection cuts: $p_{T}^{(1)}, p_{T}^{(2)}, p_{T}^{(3)}$ are the $p_{T}$ of jets ranked by their $p_{T}$. $N_{\text {jets }}$ is the number of jets in an event.

\begin{tabular}{lcccc}
\hline \hline & $p_{T}^{(1)}(\mathrm{GeV})$ & $p_{T}^{(2)}(\mathrm{GeV})$ & $p_{T}^{(3)}(\mathrm{GeV})$ & $N_{\text {jets }}$ \\
\hline Cut 1 & $\geq 50$ & $\geq 30$ & $\geq 25$ & $\geq 4$ \\
Cut 2 & $\geq 70$ & $\geq 40$ & $\geq 25$ & $\geq 4$ \\
Cut 3 & $\geq 70$ & $\geq 40$ & $\geq 25$ & $\geq 5$ \\
\hline \hline
\end{tabular}


TABLE II. Cross sections (in fb) for the signal and various SM background processes for one (zero) electron in the final state. $V$ refers to all the electroweak gauge bosons. Cuts 1-3 are as defined in Table I. In the last column, the additional cuts are as defined in Eq. (3) with $p_{T}^{\min }=140 \mathrm{GeV}$ and $\Delta R^{*}=3.2$.

\begin{tabular}{|c|c|c|c|c|c|}
\hline & Generator-level & Cut 1 & Cut 2 & Cut 3 & Cut $3+p_{T}\left(e^{-}\right) / \mathrm{MET}+\Delta R_{\max }^{\mathrm{jet}}$ \\
\hline Signal for $m_{\tilde{g}}=50 \mathrm{GeV}$ & $38(14)$ & $11.13(5.47)$ & $6.52(3.90)$ & $2.61(1.60)$ & $0.29(0.36)$ \\
\hline Signal for $m_{\tilde{g}}^{9}=70 \mathrm{GeV}$ & $12(4.7)$ & $6.78(3.33)$ & $4.23(2.48)$ & 2.33 & $0.20(0.27)$ \\
\hline QCD background & $\begin{array}{c}4 \mathrm{j}: 5270(270) \\
5 \mathrm{j}: 579(169)\end{array}$ & 3140 (350) & $1610(230)$ & $238(96)$ & $6.29(8.63)$ \\
\hline$p+e^{-} \rightarrow j+e^{-} / \nu_{e}+j+V, V \rightarrow j j$ & $230(157)$ & $131(101)$ & $88(74)$ & $0(0)$ & $0(0)$ \\
\hline$p+e^{-} \rightarrow j+e^{-} / \nu_{e}+b \bar{t} / \bar{b} t, t / \bar{t} \rightarrow b / \bar{b}+j j$ & $0.1(171)$ & $0.09(131)$ & $0.08(92)$ & $0.04(42)$ & $<0.01(1.24)$ \\
\hline$p+e^{-} \rightarrow j+e^{-} / \nu_{e}+j+h, h \rightarrow b \bar{b}$ & $1.5(7.5)$ & $1.2(6.0)$ & $0.98(4.8)$ & $0(0)$ & $0(0)$ \\
\hline$p+e^{-} \rightarrow j+e^{-} / \nu_{e}+V V, V \rightarrow j j$ & $5.2(3.6)$ & $4.4(3.2)$ & $3.6(2.8)$ & $1.9(1.5)$ & $<0.1(<0.1)$ \\
\hline$p+e^{-} \rightarrow j+e^{-} / \nu_{e}+t \bar{t}, t \bar{t} \rightarrow$ hadronic & $1.96(0.01)$ & $1.9(0.01)$ & $1.82(0.01)$ & $1.77(0.01)$ & $<0.01(<0.01)$ \\
\hline$p+e^{-} \rightarrow j+e^{-} / \nu_{e}+t \bar{t}, t \bar{t} \rightarrow$ semileptonic & $1.96(0.01)$ & $1.83(0.01)$ & $1.6(0.01)$ & $0.99(0.01)$ & $<0.01(<0.01)$ \\
\hline Total background & $\begin{array}{c}4 \mathrm{j}: 5500(435) \\
5 \mathrm{j}: 708(224)\end{array}$ & $3280(590)$ & $1710(402)$ & $242(139)$ & $6.3(9.9)$ \\
\hline
\end{tabular}

TABLE III. $\quad S / B$ and signal significance for RPV gluino signal events at the LHeC for $m_{\tilde{g}}=50,70 \mathrm{GeV}$. The signal significance with systematic error is computed using Eq. (2). Cuts 1-3 are as defined in Table I. In the last column, the additional cuts are as defined in Eq. (3) with $p_{T}^{\min }=140 \mathrm{GeV}$ and $\Delta R^{*}=3.2$. The signal significances for one- and zero-electron events, written inside the brackets in the same order, are added in quadrature to get the combined signal significance.

\begin{tabular}{lccccc}
\hline \hline & $m_{\tilde{g}}(\mathrm{GeV})$ & Cut 1 & Cut 2 & Cut 3 & Cut 3+ $p_{T}\left(e^{-}\right) / \mathrm{MET}+\Delta R_{\max }^{\text {jet }}$ \\
\hline$S / B \times 100$ & 50 & $(0.34,0.92)$ & $(0.38,0.97)$ & $(1.1,1.1)$ & $(4.6,3.6)$ \\
& 70 & $(0.21,0.56)$ & $(0.25,0.62)$ & $(0.96,0.95)$ & $(3.2,2.7)$ \\
Statistical significance, & 50 & $9.4(6.15,7.11)$ & $7.91(4.98,6.15)$ & $6.92(5.30,4.29)$ & $5.12(3.63,3.61)$ \\
$S / \sqrt{B}$ & 70 & $5.72(3.74,4.33)$ & $5.07(3.23,3.91)$ & $5.92(4.73,3.56)$ & $3.74(2.54,2.74)$ \\
Significance with 1\% & 50 & $0.98(0.34,0.92)$ & $1.03(0.38,0.96)$ & $1.53(1.05,1.11)$ & $3.83(2.84,2.56)$ \\
systematic error & 70 & $0.60(0.21,0.56)$ & $0.67(0.25,0.61)$ & $1.31(0.94,0.92)$ & $2.78(1.99,1.94)$ \\
\hline \hline
\end{tabular}

electron events after applying these cuts are as follows: 6.3 (9.9) $\mathrm{fb}$ for the background, $0.29(0.36) \mathrm{fb}$ for the signal from $m_{\tilde{g}}=50 \mathrm{GeV}, 0.20(0.27) \mathrm{fb}$ for the signal from $m_{\tilde{g}}=70 \mathrm{GeV}$. As listed in Table III, this gives the combined signal significance of $\approx 3.8 \sigma$ and $\approx 2.8 \sigma$ for $m_{\tilde{g}}=50$ and $70 \mathrm{GeV}$, respectively. The signal significance for $m_{\tilde{g}}=70 \mathrm{GeV}$ is lower than that of $m_{\tilde{g}}=50 \mathrm{GeV}$ by a factor of $\sim 0.7$. This is a combination of the production cross section for the former being less by a factor of $\sim 0.3$ (see Table II) and the $p_{T}$-based cuts being comparatively
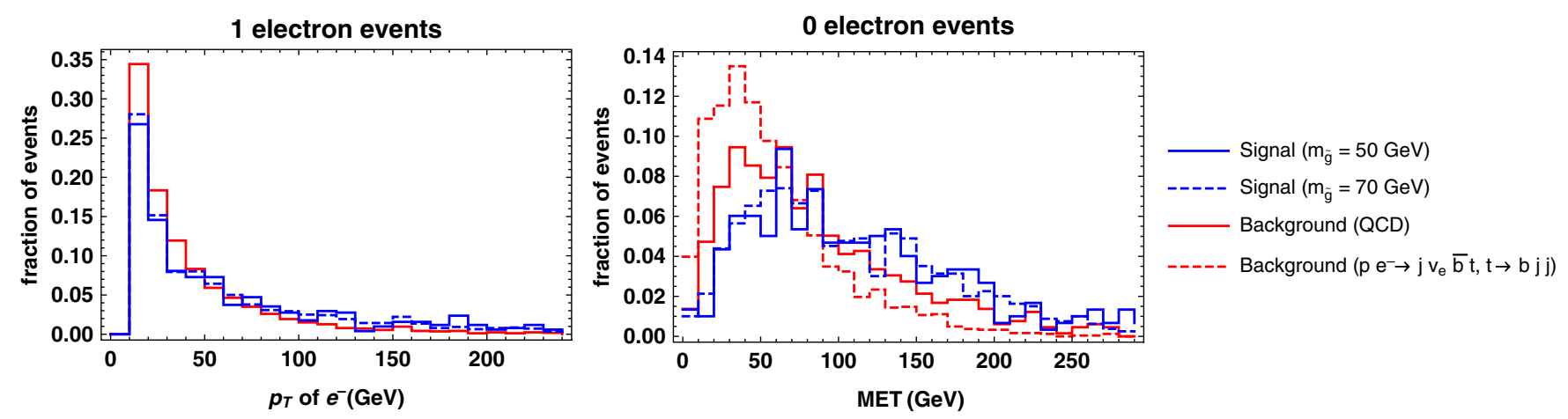

FIG. 4. Distributions for the $p_{T}$ of outgoing electrons or MET for events with one and zero electrons in the final state, respectively. These distributions are obtained after applying Cut 3 (see Table I). The blue solid and dashed lines refer to the signal from $m_{\tilde{g}}=50$ and $70 \mathrm{GeV}$, respectively. The red solid line shows the QCD background. The red dashed line is the background contribution to zero-electron events from $p+e^{-} \rightarrow j+\nu_{e}+\bar{b}+t, t \rightarrow b+j j$, which has a cross section comparable to that of the QCD processes after applying Cut 3 (see Table II). 

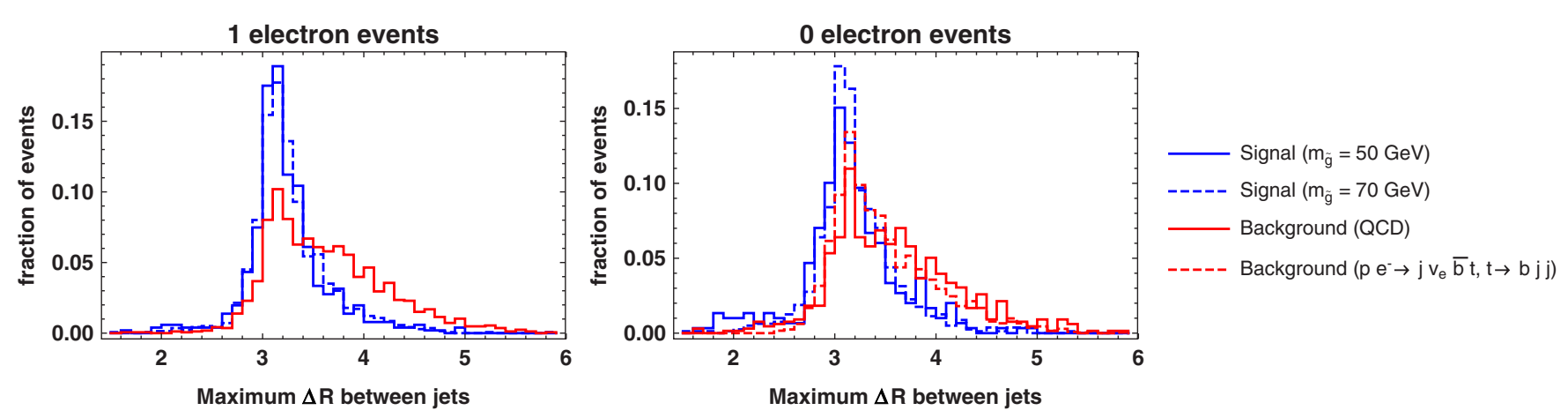

FIG. 5. Distributions for the maximum $\Delta R$ among all pairs of jets in an event. These distributions are obtained after applying Cut 3 (see Table I). The blue solid and dashed lines refer to the signal from $m_{\tilde{g}}=50$ and $70 \mathrm{GeV}$, respectively. The red solid line shows the QCD background. The red dashed line is the background contribution to zero-electron events from $p+e^{-} \rightarrow j+\nu_{e}+\bar{b}+t$, $t \rightarrow b+j j$, which has a cross section comparable to that of the QCD processes after applying Cut 3 (see Table II).

harder on the latter (see Fig. 3 and Table II). Thus, this shows that even in the presence of $1 \%$ systematic error, we can use simple cuts like Eq. (3) and reach the $2 \sigma$ exclusion limit for this signal at the LHeC. More sophisticated signal selection strategies based on jet substructure, machinelearning techniques and with more knowledge of the detector are expected to significantly enhance the prospects of signal detection.

\section{CONCLUSIONS}

RPV or stealth SUSY can give rise to gluinos with allhadronic and multijet decay with no missing energy signal. This is not subject to constraints from the standard SUSY searches at the LHC. As pointed out in Ref. [31], there is an existing gap in the search for such light gluinos with $m_{\tilde{g}} \approx 50-70 \mathrm{GeV}$. Such a BSM scenario giving a soft multijet signal is very challenging to probe at the LHC (or future hadron colliders) due to the large pileup and background. In this work, for the benchmark case of RPV SUSY, we have shown that this gap can be covered at the $\mathrm{LHeC}$. Even in the presence of $1 \%$ systematic error, using simple signal selection cuts based on the $p_{T}$ of jets, $e^{-}$and the isotropy of signal jets, the $\mathrm{LHeC}$ has the potential to observe soft multijet signals from these light RPV gluinos with $\geq 2 \sigma$ significance i.e., at the $95 \%$ confidence level.

We demonstrated that the $\mathrm{LHeC}$ has a unique sensitivity to BSM scenarios giving soft multijet signals which are very hard to probe at the $p p$ colliders, such as the RPV gluino considered here. It is likely that similar opportunities exist for hidden valley models [35-40] which can yield high-multiplicity soft hadronic signals at detectable rates. Thus, as first illustrated in Ref. [17], $e^{-} p$ colliders have a unique potential to detect BSM signals that look like hadronic noise at the $p p$ colliders thus providing a complementary discovery potential to the future $p p$ colliders. Further study is required to identify more such generic BSM scenarios which will add to the motivation for the construction of the future proposed $e^{-} p$ colliders.

\section{ACKNOWLEDGMENTS}

We thank the LHeC working group for their feedback and valuable discussions. We thank L. Delle Rose for collaboration in the early stages of this work. K. D. is supported by National Science Foundation Grant No. PHY-1620074 and the Maryland Center for Fundamental Physics. O.F. has received funding from the European Union's Horizon 2020 research and innovation program under the Marie Sklodowska-Curie Grant Agreement No. 674896 (Elusives). The research activities of D. C. are supported by a Discovery Grant from the Natural Sciences and Engineering Research Council of Canada.
[1] T. Golling et al., CERN Yellow Report No. CERN-TH2016-111, 2017.

[2] M. L. Mangano et al., CERN Yellow Report No. CERNTH-2016-112, 2017.

[3] R. Contino et al., CERN Yellow Report No. CERN-TH2016-113, 2017.
[4] J. Tang et al., arXiv:1507.03224.

[5] H. Baer, T. Barklow, K. Fujii, Y. Gao, A. Hoang, S. Kanemura, J. List, H. E. Logan, A. Nomerotski, M. Perelstein et al., arXiv:1306.6352.

[6] J. E. Brau, T. Barklow, J. Brau, K. Fujii, J. Gao, J. List, N. Walker, and K. Yokoya (ILC Parameters Joint Working 
Group), in Proceedings, Meeting of the APS Division of Particles and Fields (DPF 2015): Ann Arbor, Michigan, USA, 2015 (unpublished).

[7] M. Bicer et al. (TLEP Design Study Working Group), J. High Energy Phys. 01 (2014) 164.

[8] M. Abbrescia et al. (CEPC Study Group), arXiv:1809 .00285 .

[9] M. Abbrescia et al. (CEPC Study Group), arXiv:1811 .10545 .

[10] M. Aicheler, P. Burrows, M. Draper, T. Garvey, P. Lebrun, K. Peach, N. Phinney, H. Schmickler, D. Schulte, and N. Toge, CERN Yellow Reports No. CERN-2012-007, No. SLAC-R-985, No. KEK-Report-2012-1, No. PSI-1201, and No. JAI-2012-001, 2012.

[11] D. M. South (H1 Collaboration), Proc. Sci. ICHEP2012 (2013) 141.

[12] V. M. Abazov et al. (D0 Collaboration), Phys. Rev. D 84, 071104 (2011).

[13] M. Klein, in Proceedings of the 17th International Workshop on Deep-Inelastic Scattering and Related Subjects (DIS 2009): Madrid, Spain, 2009 (2009).

[14] J. L. Abelleira Fernandez et al. (LHeC Study Group), J. Phys. G 39, 075001 (2012).

[15] O. Bruening and M. Klein, Mod. Phys. Lett. A 28, 1330011 (2013).

[16] F. Zimmermann, M. Benedikt, D. Schulte, and J. Wenninger, in Proceedings of the 5th International Particle Accelerator Conference (IPAC 2014): Dresden, Germany, 2014 (2014), p. MOXAA01.

[17] D. Curtin, K. Deshpande, O. Fischer, and J. Zurita, J. High Energy Phys. 07 (2018) 024.

[18] D. Curtin et al., arXiv:1806.07396.

[19] S. P. Martin, Adv. Ser. Dir. High Energy Phys. 21, 1 (2010).

[20] M. Aaboud et al. (ATLAS Collaboration), Phys. Rev. D 97, 112001 (2018).
[21] M. Aaboud et al. (ATLAS Collaboration), Phys. Rev. D 99, 012009 (2019).

[22] J. A. Evans, Y. Kats, D. Shih, and M. J. Strassler, J. High Energy Phys. 07 (2014) 101.

[23] R. Barbier et al., Phys. Rep. 420, 1 (2005).

[24] J. Fan, M. Reece, and J. T. Ruderman, J. High Energy Phys. 11 (2011) 012.

[25] D. E. Kaplan and M. D. Schwartz, Phys. Rev. Lett. 101, 022002 (2008).

[26] J. Llorente and B.P. Nachman, Nucl. Phys. B936, 106 (2018).

[27] G. Aad et al. (ATLAS Collaboration), J. High Energy Phys. 12 (2012) 086.

[28] S. Chatrchyan et al. (CMS Collaboration), Phys. Rev. Lett. 107, 101801 (2011).

[29] S. Chatrchyan et al. (CMS Collaboration), Phys. Lett. B 718, 329 (2012).

[30] T. Aaltonen et al. (CDF Collaboration), Phys. Rev. Lett. 107, 042001 (2011).

[31] J. A. Evans and D. Mckeen, arXiv:1803.01880.

[32] G. Azuelos, M. D’Onofrio, O. Fischer, and J. Zurita, in 26th International Workshop on Deep Inelastic Scattering and Related Subjects (DIS 2018) Port Island, Kobe, Japan, 2018 (2018).

[33] J. Alwall, R. Frederix, S. Frixione, V. Hirschi, F. Maltoni, O. Mattelaer, H. S. Shao, T. Stelzer, P. Torrielli, and M. Zaro, J. High Energy Phys. 07 (2014) 079.

[34] B. Fuks, Int. J. Mod. Phys. A 27, 1230007 (2012).

[35] M. J. Strassler and K. M. Zurek, Phys. Lett. B 651, 374 (2007).

[36] M. J. Strassler and K. M. Zurek, Phys. Lett. B 661, 263 (2008).

[37] M. J. Strassler, arXiv:hep-ph/0607160.

[38] T. Han, Z. Si, K. M. Zurek, and M. J. Strassler, J. High Energy Phys. 07 (2008) 008.

[39] M. J. Strassler, arXiv:0801.0629.

[40] M. J. Strassler, arXiv:0806.2385. 